\title{
The immunomodulating role of probiotics in the prevention and treatment of oral diseases
}

\author{
MARTA KAŹMIERCZYK-WINCIOREK, MAEGORZATA NĘDZI-GÓRA, \\ SYLWIA MAEGORZATA SEOTWIŃSKA
}

Department of Oral Hygiene, Medical University of Warsaw, Warsaw, Poland

\begin{abstract}
Probiotics are defined as non-pathogenic live microorganisms which, when administered in appropriate amounts, are beneficial to the health of the host. Currently, the group of probiotic microorganisms includes bacteria that produce lactic acid of the genera Lactobacillus and Bifidobacterium, belonging to the so-called LAB (lactic acid bacteria) groups, and the yeast Saccharomyces boulardii. Probiotics have a protective role in Candida spp. oral infection and especially colonization. Lactobacillus limits the progression of chronic periodontitis by inhibiting the secretory activity of Th17 lymphocytes, which in the pathogenesis of this disease are responsible for an excessive cytokine response causing adverse changes in periodontal tissues. A meta-analysis of the results of studies on the clinical evaluation of the effectiveness of probiotics in the treatment of gingivitis showed that the use of probiotics improved the condition of the gums in the course of the therapeutic process. Regular use of probiotics during orthodontic treatment significantly reduces the number of bacteria from the Streptococcus mutans group in the patient's saliva and significantly inhibits the expression of inflammatory mediators and an excessive immune response. The main mechanisms of the action of probiotics, such as elimination and inhibition of the growth of pathogenic microorganisms through competition for receptor sites and the secretion of metabolites with antibacterial activity, as well as the stimulation of specific and non-specific immune responses by activating T lymphocytes, and stimulating the production of cytokines, make it possible to also effectively use pro-health bacteria in oral diseases.
\end{abstract}

Key words: probiotics, oral diseases, therapy.

(Cent Eur J Immunol 2021; 46 (1): 99-104)

\section{Introduction}

Pro-health behaviors related to a healthy lifestyle and a well-balanced diet play a special role in regulating the composition of the human intestinal microflora. Functional food has an important place in this process [1-4], and is defined as all products of natural origin with a high pro-health and nutritional value that can be modified or enriched, but only with natural substances, positively influencing human health and development. Research on the influence of nutrition on the human body has been conducted for many years and is multidirectional $[5,6]$. Almost all possible food ingredients and their effect on metabolism are assessed. This research aims to develop such a diet composition that will constitute food as another tool in the prevention of many diseases, especially lifestyle diseases, and one that perhaps may supplement therapeutic management [7-9]. Probiotic food enriched with live cultures of lactic acid bacteria, for which the natural carriers are consumed grain and dairy products, as well as fruit preserves, fit perfectly into this research stream. Probiotic bacteria occupy a unique place in human nutrition, along with prebiotics and synbiotics [10]. Their beneficial effect on the entire gastrointestinal tract and the general health of the human body has been proven many times.

\section{Probiotics, prebiotics, synbiotics, and psychobiotics}

Probiotics are defined as non-pathogenic live microorganisms which, when administered in appropriate amounts, are beneficial to the health of the host $[5,6]$. They have the ability to produce antibacterial compounds, counteract the adhesion of pathogenic microorganisms, and modulate immunological functions. The bacteriostatic and bactericidal activity of probiotics is based on the production of metabolites with an antagonistic effect against pathogens, competition in intestinal wall colonization, and stimulation of the immune system to intensify the production of antibodies. Lactobacillus shows great ability to colonize human mucosa, especially in the digestive tract. The ba-

Correspondence: Prof. Sylwia Małgorzata Słotwińska, Department of Oral Hygiene, Medical University of Warsaw, Warsaw, Poland, e-mail: sylwia.slotwinska@wum.edu.pl

Submitted: 25.11.2020; Accepted: 31.12.2020 
sic principle in the use of probiotics is based on the assumption that a probiotic cannot be harmful to the human body with a properly functioning immune system, without the characteristics of immune disorders. Probiotic strains must meet the GRAS (generally recognized as safe) standard, and the safety of their use must be confirmed by both in vitro and in vivo studies $[5,6,10]$.

Currently, the group of probiotic microorganisms includes bacteria that produce lactic acid of the genera Lactobacillus (L. acidophilus, L. plantarum, L. reuteri, L. rhamnosus, L. casei) and Bifidobacterium (B. animalis, $B$. bifidum, B. breve), belonging to the so-called LAB (lactic acid bacteria) groups, and the yeast Saccharomyces boulardii.

Prebiotics are a constituent of food that are resistant to the action of gastrointestinal enzymes, are not digestible, and are not absorbed by the intestinal mucosa. They are referred to as food for the intestines because they have an extremely beneficial effect on the host by selectively stimulating the growth and/or metabolic activity of one or a limited number of bacteria that make up the microflora of the gastrointestinal tract, especially in the colon [11]. Unlike probiotics, they do not contain any microorganisms, only stimulating substances. A prebiotic can be a natural dietary component, e.g. starch or dietary fiber, as well as dietary supplements with a health-promoting effect. Very often, prebiotics are added to food to improve the nutritional and health value, e.g. fructo-oligosaccharides, lactulose, galactose derivatives. They are a special medium for probiotics, strengthening their metabolism and accelerating colony development. The main task of prebiotics is to stimulate the growth of lactic acid bacteria and inhibit the activity of harmful intestinal microflora. Additionally, they significantly reduce the so-called bad low-density lipoprotein (LDL) cholesterol in the blood, stimulating the immune system, reducing the risk of colon cancer and inflammatory bowel diseases such as Crohn's disease and ulcerative colitis, and help maintain an appropriate $\mathrm{pH}$ value in the gut. They also prevent neoplastic reactions and support the treatment of dental caries and are useful in reducing the effects of lactose intolerance [12-14].

A synbiotic is a combination of a probiotic and a prebiotic working together to restore normal gut microflora. Such synergistic action contributes to the reduction of the concentration of undesirable metabolites in the body, inactivation of nitrosamines and carcinogenic substances, and also prevents putrefactive processes in the intestines as well as constipation and diarrhea of various etiologies [13]. Synbiotics reduce too high levels of cholesterol and lower blood pressure [14]. Moreover, they are used in complementary therapy in the treatment of patients with gastrointestinal diseases [15], also improving the absorption of calcium, magnesium, and phosphorus [16].

It is also worth mentioning psychobiotics - bacteria that affect the gut-brain axis, which has a positive effect on human emotional states [17]. They include both Lactobacillus and Bifidobacterium strains. Their main activity is the secretion of the GABA neurotransmitter ( $\gamma$-aminobutyric acid), which acts on the brain through the vagus nerve. Psychobiotics have been used in the treatment of mental diseases, depression, and irritable bowel syndrome as well as in states of chronic fatigue. Research on this issue began at the beginning of the $21^{\text {st }}$ century, and the published results indicate a significant role of probiotic bacteria in improving the mental condition of both experimental animals and volunteers who took part in the experiment [18].

\section{The mechanism of action of probiotics in the oral cavity}

The mechanism of action of probiotic bacteria in the oral cavity is analogous to that of the intestine. It consists mainly of the production of bacteriocins and stimulation of the local immune response. Additionally, probiotics in the oral cavity change the composition of the ecological niche of microorganisms by competing with pathogenic bacteria for mucosal receptors and nutrients and reduce the excessive inflammatory response [19-22]. Bacteria commensally living in the environment of the human organism developed the property of modulating the host's immune response. They can influence the production of cytokines and antibodies and increase the pool of regulatory T lymphocytes [23]. Lactobacillus strains have the ability to induce cytokine synthesis by the inflammatory cells. They affect the production of both pro-inflammatory cytokines [tumor necrosis factor (TNF) 1, interleukin (IL)-12] and cytokines that inhibit inflammatory processes (IL-10). Additionally, they induce the synthesis of antibodies of the sIgA class - secretory immunoglobulin A. Probiotic microorganisms modify the receptors for bacterial toxins, which may indirectly reduce inflammation. They obtain permanent colonization by the mechanism of adherence to receptors located in the cells of the mucosa. The structure of the cell membrane surface of the genus Lactobacillus enables them to adhere and create a biofilm on various surfaces [24]. Colonies are very persistent and stable because cell membranes contain numerous elements such as capsular polysaccharides, lipoteichoic acids, teichoic acids, surface proteins, and lipoproteins, which contribute to hydrophobicity and the ability to strongly auto-aggregate strains of the genus Lactobacillus [25, 26]. In the first stage of adherence, when the distance between bacterial cells and the occupied surface area is large, the most important role is played by the physical intermolecular interactions related to van der Waal's gravitational forces and hydrophobic and thermodynamic mechanisms. This allows the cell to come closer to the place that is supposed to be inhabited. The next step occurs when the distance between the cell 
and the surface is less than $1.5 \mathrm{~nm}$. Specific connections are made between the inhabited surface with the appropriate receptors and adhesive proteins on the bacterial surface $[27,28]$.

\section{Probiotics and oral candidiasis}

The influence of probiotics on inflammation of the oral cavity has been thoroughly examined in the course of fungal etiology. Oral candidiasis is a serious and constantly growing health problem in modern society, mainly due to the aging of the population and the increasing incidence of chronic diseases, as well as the constant introduction of new drugs, especially antibiotics. Candida is one of the microorganisms that have the ability to colonize various places in the human body. Localization concerns the oral cavity and the skin, gastrointestinal tract, genitourinary system, and respiratory tract. Under certain unfavorable conditions, Candida can change from being commensally harmless to an unfavorable pathogenic form leading to the development of a dangerous infection. The positive characteristics of probiotic bacteria and their ability to immunomodulate allow for the elimination of the unfavorable growth of fungal colonies in the oral cavity, which has been confirmed in many experimental and clinical studies that demonstrated both the prophylactic properties of these microorganisms and their therapeutic effect. Mundula et al. described the results obtained from a meta-analysis and concluded that probiotics have a protective role in Candida spp. infection and especially colonization. This study systematically reviewed the effects of the intake of probiotics on oral candidiasis and concluded that this treatment had a beneficial effect on reducing oral Candida spp. counts [23].

The antifungal activity of probiotics has been successfully applied in the treatment of oral candidiasis in experimental animals with an immunological deficiency. Wagner et al. stated that the administration of probiotics could be a prophylactic and therapeutic strategy for mucosal candidiasis. They demonstrated that the presence of four strains of bacteria (L. acidophilus, L. reuteri, L. casei GG, and B. animalis) in the gastro-intestinal tract of immunodeficient mice reduced the number of Candida albicans cells, as well as the incidence and severity of mucosal and systemic candidiasis, prolonging their survival [29]. Of particular interest and importance are the clinical observations concerning patients with head and neck cancer undergoing radiotherapy. Probiotic bacteria were administered to these patients in rinse form and an effective reduction of Candida spp. strains in the mouth was achieved. In addition to reducing the number of colonies of the Candida albicans species, after the use of probiotics in the rinse, a significant decrease in Candida glabrata and Candida tropicalis colonies was observed as well as a significant improvement in the clinical condition of the oral mucosa compared to the control group [30].

\section{Probiotics and periodontal diseases}

Gum and periodontium diseases are another group of diseases in which attempts are made to use probiotics as supplementation in basic treatment. Inadequate oral hygiene and the accumulation of bacterial biofilm promote the formation of local inflammation, which causes gingivitis and leads to the development of chronic periodontitis - a disease that induces the uncontrolled production of pro-inflammatory cytokines and the development of numerous disorders in the local immune response [31-33]. The relationship of periodontitis with many systemic and chronic diseases has been proven. Thus, periodontitis is a local bacterial disease with very large systemic implications. The basic characteristic of the selected strains of probiotic bacteria is the peripheral reduction of other populations of microorganisms responsible for the occurrence of diseases, in the etiopathogenesis of which the microbiological factor plays a major role. It is believed that the use of probiotics in the prevention and treatment of gingivitis and periodontitis may be of great importance. Lactobacillus limits the progression of chronic periodontitis by inhibiting the secretory activity of Th17 lymphocytes, which in the pathogenesis of this disease are responsible for an excessive cytokine response causing adverse changes in periodontal tissues [34]. A meta-analysis of the results of studies on the clinical evaluation of the effectiveness of probiotics in the treatment of gingivitis showed that the use of probiotics improved the condition of the gums in the course of the therapeutic process. The mean percentages of the bleeding on the probing (BOP) index ranged from $11.87 \%$ to $21.7 \%$ in the probiotic supplementation group, compared to the placebo control group: from $15 \%$ to $33 \%$. It is worth noting that in the assessment of other parameters of the gingival condition - the plaque index (PI) and the gingival index (GI) - the use of the probiotic Lactobacillus reuteri in the treatment of gingivitis showed no significant differences between the probiotic and placebo groups [35]. Highly intriguing results were obtained by Soares et al., who assessed the effect of administration of probiotics as an adjunct treatment in people with advanced periodontitis and concomitant halitosis. Sixty patients with severe periodontal disease were randomly divided into two groups and underwent intensive single-session periodontal treatment and were consecutively ordered to take the preparation with a probiotic or placebo for ninety days. The test group received Lactobacillus reuteri, Lactobacillus salivarius, and Lactobacillus acidophilus, while the control group received a placebo with xylitol. After 90 days of observation, it was found that, in the test group, the depth of periodontal pockets was shallower and the level of connective tissue attachment was reduced, as was the BOP index. Significant neutralization of halitosis in the probiotic group compared to the placebo group was also found [36]. 
Albuquerque-Souza et al. assessed the effect of probiotics Lactobacillus and Bifidobacterium on the immune response of gingival epithelial cells in the event of an invasion by bacteria of the species Porphyromonas gingivalis. In the experiment, fixed human gingival epithelial cells (GECs) (OBA-9) were infected with live bacteria of the Porphyromonas gingivalis species and simultaneously with one of the twelve tested probiotic strains. Porphyromonas gingivalis was able to adhere to and invade OBA-9 cells, with significant loss of cell viability, increase in the levels of TNF- $\alpha$ and IL-1 $\beta$, and upregulation of TLR4. Most probiotics maintained OBA-9 viability and reduced pathogens adhesion and invasion. Furthermore, probiotics were able to adhere to GECs, which was enhanced for most strains in the presence of Porphyromonas gingivalis. The synthesis of IL- $1 \beta$ and TNF- $\alpha$ by Porphyromonas gingivalis in challenged GECs was reduced in co-culture with most of the tested probiotics, whereas the secretion of CXCL8 increased, and TLR4 was downregulated. Co-infection with probiotic microorganisms caused a decrease in the virulence of pathogenic bacteria and a weakening of their adhesive capacity. The applied probiotics changed the interaction between epithelial cells and bacteria of the species Porphyromonas gingivalis, which clearly indicates the immense usefulness of probiotics in the prevention and treatment of gingivitis and periodontitis [37]. Caradoso et al. examined the effectiveness of systemic administration of a probiotic, Bifidobacterium animalis, subsp. lactis HN019, to laboratory rats. Systemic administration of HN019 resulted in a reduction in bone mass loss, a decrease in the number of inflammatory mediators, and an increase in the percentage of colonies of physiological microflora in experimental animals [38].

The presented research results indicate the beneficial effects of probiotics both in the prevention and treatment of gingivitis and periodontitis. During follow-up examinations, clinical improvement in the diagnosis of gingival bleeding during probing was demonstrated as well as reduction of depth of the gingival pockets and in the level of connective tissue attachment. In addition, probiotics, by producing hydrogen peroxide, can be antagonistic in periopathogenic microorganisms, which significantly reduces the progression of gingivitis and chronic periodontitis.

\section{Probiotics and orthodontic treatment}

During orthodontic treatment, in some patients, it is very common to observe a gradual deterioration of oral hygiene. This is mainly due to the inability to thoroughly and properly clean the tooth surface, especially after the insertion of a fixed appliance. In the patient's oral cavity, the accumulation of dental bacterial biofilm and the growth of colonies of pathogenic microorganisms primarily occur. As a consequence, gingivitis is characterized by reddening of the marginal gingiva, swelling and enlargement of the interdental papillae, and bleeding from the gums. In the process of the first phase of treatment, during the correct alignment of the teeth, the conditions for maintaining proper hygiene on the surfaces and interdental spaces are significantly improved, which reduces the number of pathogenic bacteria. It is believed that one of the reasons for the reduction in the number of pathogenic microbe colonies during treatment with metal braces may be corrosion, during which metal ions that are toxic to microorganisms are released [39]. The occurrence of gingivitis in orthodontically treated patients may lead to the development of periodontitis and, consequently, the loss of connective tissue attachment and bone loss of the alveolar ridge, resulting in increased tooth mobility, which is usually the cause of poor prognosis and treatment failure. In addition to stringent recommendations to maintain a strict oral hygiene regime, usually in such cases chlorhexidine rinses are recommended as an adjunct. However, due to the occurrence of side-effects, it is not recommended for chronic use for longer than 14 days. Due to the long process of orthodontic treatment, probiotics may be a better solution. Probiotic bacteria, such as Lactobacillus strains contained in food supplements, can restore disturbed microbiological balance and eliminate dysbiosis, which is unfavorable for the organism. Regular use of probiotics during orthodontic treatment significantly reduces the number of bacteria from the Streptococcus mutans group in the patient's saliva and significantly inhibits the expression of inflammatory mediators and an excessive immune response. Studies have shown that better results are obtained with preparations for topical use than with systemic preparations [40-42].

Probiotics, prebiotics, and synbiotics are used with great success in many diseases of the gastrointestinal tract and disorders related to dyspeptic conditions $[4,9,11]$. Additionally, they are used in allergies and autoimmune diseases as well as in the treatment of overweight and obesity, and in eating disorders such as anorexia and bulimia. There are also attempts to use probiotics in the prevention of recurrent infections and cancer [1-3, 43]. Recently, the possibility of using probiotics in the prevention and treatment of oral diseases including tooth caries, oral candidiasis, halitosis as well as in gingivitis and periodontitis has also been investigated [19, 23, 29, 30, 36, 44, 45]. The main mechanisms of the action of probiotics, such as the elimination and inhibition of the growth of pathogenic microorganisms through competition for receptor sites and the secretion of metabolites with antibacterial activity, as well as the stimulation of specific and non-specific immune responses by activating $\mathrm{T}$ lymphocytes, and stimulating the production of cytokines, make it possible to also effectively use pro-health bacteria in oral and dental diseases [19-21]. Reduction of Candida and Streptococcus mutans strains in the oral cavity, reducing the number of periopathogenic bacteria colonies and inhibiting the secretion of inflammatory mediators, as well as improving the 
condition of the oral mucosa and the condition of the periodontium, have a significant impact on the general health of the human body. Therefore, the need to conduct experimental and clinical studies on the possibilities of using microorganisms present in food products and those that are part of the natural human microbiome, with particular emphasis on the environment of the digestive tract and microorganisms living in the oral cavity, is extremely important.

\section{The authors declare no conflict of interest.}

\section{References}

1. Cross ML, Stevenson LM, Gill HS (2001): Anti-allergy properties of fermented foods: an important immunoregulatory mechanism of lactic acid bacteria? Int Immunopharmacol 1: 891-901.

2. Kirjavainen PV, Apostolou E, Salminen SJ, Isolauri E (1999): New aspects of probiotics - a novel approach in the management of food allergy. Allergy 54: 909-915.

3. Majamaa H, Isolauri E (1997): Probiotics: a novel approach in the management of food allergy. J Allergy Clin Immunol 99: 179-185.

4. Salminen S, Bouley C, Boutron-Ruault MC, et al. (1998): Functional food science and gastrointestinal physiology and function. Br J Nutr 80: 147-171.

5. Probiotics in food: health and nutritional properties and guidelines for evaluation. FAO, Food and Nutrition. Paper 85. FAO/WHO. Rome 2006.

6. Fuller R (1991): Probiotics in human medicine. Gut 32: 439442.

7. Nur'aini FD, Rahayu S, Rifa'i M (2019): Anti-inflammatory activity of elicited soybean (Glycine max) extract on Balb/C mice (Mus musculus) with high-fat and -fructose diet. Cent Eur J Immunol 44: 7-14.

8. Kalicki B, Lewicka A, Jęderka K, et al. (2019): Vitamin B6 improves blood parameters in rats fed a protein-deficient diet and subjected to moderate, long-term exercise. Cent Eur J Immunol 44: 23-32.

9. Terpou A, Papadaki A, Lappa I, et al. (2019): Probiotics in food systems: significance and emerging strategies towards improved viability and delivery of enhanced beneficial value. Nutrients 13; 11: 1591.

10. Ouwehand AC, Kirjavainen PV, Shortt C, Salminen S (1999): Probiotics: mechanisms and established effects. Int Dairy $\mathbf{J}$ 9: 43-52.

11. Gibson GR, Roberfroid MB (1995): Dietary modulation of the human colonic microbiota: introducing the concept of prebiotics. J Nutr 125: 1401-1412.

12. Jakubczyk E, Kosikowska M (2000): Nowa generacja mlecznych produktów fermentowanych $\mathrm{z}$ udziałem probiotyków i prebiotyków, produkty synbiotyczne. Przegl Mlecz 12: 397-400.

13. Mojka K (2014): Probiotyki, prebiotyki i synbiotyki - charakterystyka i funkcje. Probl Hig Epidemiol 95: 541-549.

14. Socha P, Stolarczyk M, Socha J (2002): Wpływ probiotyków i prebiotyków na gospodarkę lipidową. Pediatr Współcz Gastroenterol Hepat Żyw Dziecka 4: 85-88.

15. Pathmakanthan S, Walsh M, Bengmark S, et al. (2002): Efficacy and tolerability treating acute distal ulcerative colitis with synbiotic enemas: a pilot trial. GUT 51. A307.
16. Perez- Conesa D , Lopez G, Abellan P, et al. (2006): Bioavailability of calcium, magnesium and phosphorus in rats fed probiotic, prebiotic and synbiotic powder follow-up infant formulas and their effect on physiological and nutritional parameters. J Sci Food AGR 86: 2327-2336.

17. Dinan TG, Stanton C, Cryan JF (2013): Psychobiotics: a novel class of psychotropic. Biol Psychiatry 74: 720-726.

18. Messaoudi M, Violle N, Bisson JF, et al. (2011): Beneficial psychological effects of a probiotic formulation (Lactobacillus helveticus R0052 and Bifidobacterium longum R0175) in healthy human volunteers. Gut Microbes 2: 256-261.

19. Laleman I, Teughels W (2015): Probiotics in the dental practice: a review. Quintessence Int 46: 255-264.

20. Teughels W, Van Essche M, Sliepen I, Quirynen M (2008): Probiotics and oral healthcare. Periodontol 2000 48: 111-147.

21. Zacro MF, Vess TJ, Ginsburg GS (2012): The oral microbiome in health and disease and the potential impact on personalized dental medicine. Oral Dis 18: 109-120.

22. Haukioja A (2010): Probiotics and oral health. Eur J Dent 4: 348-355.

23. Mundula T, Ricci F, Barbetta B, et al. (2019): Effect of probiotics on oral candidiasis: a systematic review and meta-analysis. Nutrients 11: 2449.

24. Janczarek M, Bachanek T, Mazur E, Chałas R (2016): The role of probiotics in prevention of oral diseases. Postepy Hig 70: 850-857.

25. Sengupta R, Altermann E, Anderson RC, et al. (2013): The role of cel surface architecture of Lactobacilli in host - microbe interactions in the gastrointestinal tract. Mediat Inflamm 2013: 237921.

26. Tuo Y, Yu H, Ai L, et al. (2013): Aggregation and adhesion properties of 22 Lactobacillus strains. J Dairy Sci 96: 42524257.

27. Percival SL, Malic S, Cruz H, Williams DW (2011): Introduction to biofilms. In: Biofilms and Veterinary Medicine. Percival SL, Knottenbelt D, Cochrane C (Eds.). Springer Series on Biofilms 6: 41-68.

28. Flemming HC, Wingender J (2010): The biofilm matrix. Nat Rev Microbiol 8: 623-633.

29. Wagner RD, Pierson C, Warner T, et al. (1997): Biotherapeutic effects of probiotic bacteria on candidiasis in immunodeficient mice. Infect Immun 65: 4165-4172.

30. Doppalapudi R, Vundavalli S, Prabhat MP (2020): Effect of probiotic bacteria on oral Candida in head and neck radiotherapy patients: A randomized clinical trial. J Cancer Res Ther 16: 470-477.

31. Yan X, Yuan Z, Bian Y, et al. (2020): Uncoupling protein-2 regulates M1 macrophage infiltration of gingiva with periodontitis. Cent Eur J Immunol 2020; 45: 9-21.

32. Yilmaz M, Kasnak G, Polat NG, et al. (2019): Pathogen profile and MMP-3 levels in areas with varied attachment loss in generalized aggressive and chronic periodontitis. Cent Eur J Immunol 44: 440-446.

33. Kaczyński T, Wroński J, Głuszko P, et al. (2019): Salivary interleukin 6, interleukin 8, interleukin 17A, and tumour necrosis factor $\alpha$ levels in patients with periodontitis and rheumatoid arthritis Cent Eur J Immunol 44: 269-276.

34. Liu CF, Tseng KC, Chiang SS, et al. (2011): Immunomodulatory and antioxidant potential of Lactobacillus exopolysaccharides. J Sci Food Agric 91: 2284-2291.

35. Akram Z, Shafgat SS, Aati S, et al. (2020): Clinical efficacy of probiotics in the treatment of gingivitis: A systematic review and metaanalysis. Aust Dent J 65: 12-20. 
36. Soares LG, Carvalho EB, Tinoco EMB (2019): Clinical effect of Lactobacillus on the treatment of severe periodontitis and halitosis: A double-blinded, placebo-controlled, randomized clinical trial. Am J Dent 32: 9-13.

37. Albuquerque-Souza E, Balzarini D, Ando-Suguimoto ES, et al. (2019): Probiotics alter the immune response of gingival epithelial cells challenged by Porphyromonas gingivalis. J Periodontal Res 54: 115-127.

38. Caradoso RS, Messora MR, Silva PHF, et al. (2020): Effects of Bifidobacterium animalis subsp. lactis HN019 on ligature-induced periodontitis in rats with experimental rheumatoid arthritis. Benef Microbes 11: 33-46.

39. Hepyukselen BG, Cesur MG (2019): Comparison of the microbial flora from different orthodontic archwires using a cultivation method and PCR: A prospective study. Orthod Craniofac Res 22: 354-360.

40. Alp S, Baka ZM (2018): Effects of probiotics on salivary Streptecoccus mutans and Lactobacillus levels in orthodontic patients. Am J Orthod Dentofacial Orthop 154: 517-523.

41. Lucchese A, Bondemark L, Marcolina M, Manuelli M (2018): Changes in oral microbiota due to orthodontic appliances: a systematic review. J Oral Microbiol 10: 1476645.

42. Jose JE, Padmanabhan S, Chitharanjan AB (2013): Systemic consumption of probiotic curd and use of probiotic toothpaste to reduce Streptococcus mutans in plaque around orthodontic brackets. Am J Orthod Dentofacial Orthop 144: 67-72.

43. Fukui M, Fujino T, Tsutsui K, et al. (2001): The tumor-preventing effect of a mixture of several lactic acid bacteria on 1,2-dimethylhydrazine-induced colon carcinogenesis in mice. Oncol Rep 8: 1073-1078.

44. Gruner D, Paris S, Schwendicke F (2016): Probiotics for managing caries and periodontitis: systemic review and meta-analysis. J Dent 48: 16-25.

45. Nędzi-Góra M, Wróblewska M, Górska R (2020): The effect of Lactobacillus salivarius SGL03 on clinical and microbiological parameters in periodontal patients. Pol J Microbiol 69: 441-451. 